\title{
Premiers pas dans la neurobiologie du cerveau qui pense
}

La neurobiologie est-elle en passe de s'attaquer aux phénomènes qui conduisent à la pensée consciente? C'est en tout cas le thème autour duquel s'articulent trois textes récents publiés dans Nature. Celui qui pose la question le plus abruptement est Antonio Damasio (Univ. Iowa, USA) [1] dans un commentaire sur les deux autres communications qu'il associe dans une même question: "Où trouve-t-on les corrélats neurophysiologiques de la conscience? " A première vue, pourtant, il peut être difficile à des non-spécialistes de voir dans la publication de Francis Crick et Christof Koch (Inst. Salk et CalTech, CA, USA) [2] et dans celle de Roger Tootell et al. de l'équipe de Bruce Rosen (MGH, MA, USA) [3] ce qui stimule un tel enthousiasme. Il n'en est pas moins vrai que, sans jamais que les auteurs le disent comme le souligne Damasio, c'est bien de cela dont il s'agit.

Francis Crick est depuis longtemps passé de la molécule d'ADN au cerveau et il a produit, dans les années écoulées, plusieurs réflexions sur son fonctionnement à partir, non pas directement d'études expérimentales personnelles, mais d'analyses approfondies du travail des autres. Comme l'indique l'introduction de ce dernier article, le fait de poser une question de façon parfaitement théorique permet éventuellement de dérouler une suite logique et d'aboutir à des conclusions opératoires pour de futures recherches. Francis Crick et Christoff Koch se livrent cette fois à ce travail autour d'une question qui, dans son énoncé primaire, est parfaitement réductionniste: ce que nous voyons a-t-il un lien direct avec l'acti- visuel primaire, c'est-à-dire dans l'aire (dite Vl) du cortex cérébral qui reçoit directement les messages provenant de la rétine (après relais dans le thalamus) ? La question n'est pas aussi triviale qu'il y paraît et la réponse - négative - à laquelle parviennent les auteurs est, tout compte fait, parfaitement recevable. Les neurones de l'aire V1 sont certes directement impliqués dans la transmission de ces messages. Toutefois, les caractéristiques anatomiques et physiologiques des neurones de Vl ne leur permettent, argumentent Crick et Koch, ni de produire des messages correspondant aux besoins de la vision consciente, ni de les adresser aux endroits où s'effectue cette "prise de conscience". En ce qui concerne le premier point, il est facile aux auteurs de montrer, par exemple, que les neurones de V1, strictement monoculaires, ne peuvent créer directement les sensations visuelles binoculaires. On sait depuis trente ans, et les travaux de Hubel et Wiesel en particulier, que les neurones rétiniens codent de façon très primaire le monde visuel et que le cortex cérébral recrée les images le long d'une chaîne dont les neurones de Vl ne sont que les premiers maillons. En ce qui concerne l'anatomie, il est là aussi bien connu que les neurones de V1 ne projettent que vers des zones voisines du cortex occipital et temporal (que la nomenclature la plus utilisée appelle V2, ..., V5). Cela exclut notamment des projections directes vers les régions du cortex frontal dans lesquelles s'opère, très vraisemblablement, la reconstruction opératoire des sensations visuelles. Cela serait assez banal et peu concluant quant à "l'exclusion" de V1 de la construction des images mentales si Crick et Koch n'apportaient pas, en complément, l'argument de certaines illusions optiques et de leur absence apparent de lien avec les neurones de Vl.

Or, heureux effet du hasard, probablement, l'article de Tootell et al. présente justement des arguments tout à fait concluants en faveur d'un lien unissant l'activité de neurones corticaux et l'existence d'un de ces phénomènes mentaux fondés sur une illusion d'optique. Ces auteurs ont utilisé la technique d'imagerie par résonance magnétique nucléaire fonctionnelle (voir $\mathrm{m} / \mathrm{s}$ vol $8, n^{\circ} 110$, p. 1118) pour identifier les zones corticales actives lors de la production d'un phénomène psychophysique d'illusion optique appelé "effet de la chute d'eau». Lorsque l'on regarde un objet en mouvement continu dans une direction unique, l'arrêt du mouvement provoque une illusion de mouvement dans le sens opposé Ce post-effet, qui dans le test expérimental utilisé, fondé sur une stimulation de plusieurs dizaines de secondes formé par l'élargissement ou le rétrécissement de cercles concentriques dure de dix à vingt secondes, est accompagné, de façon quasi spécifique (du moins fortement prédominante) par une activité neuronale dans l'aire corticale V5 (dite aussi MT) que l'on a identifiée depuis des années comme l'aire dans laquelle est codé le mouvement de stimuli visuels. Ainsi, c'est vraisemblablement l'activité des neurones de l'aire V5 qui crée, à elle seule, l'image mentale appréciée comme un mouvement de sens opposé à celui du stimulus. L'activité des neurones de V5 - qui répondent, eux, au critè- 
re anatomique de (.rick et Koch puisqu'ils projettent vers les régions frontales - est donc corrélée à la création cérébrale d'une image, d'un "phénomène conscient » dit Damasio.

La «pensée " est bien plus complexe que cela, ajoute Damasio dans son commentaire, en soulignant à juste titre que les sujets sont parfaitement capables de faire, notamment, la différence entre la sensation liée au mouvement réel et le post-effet, ce qui indique que l'image mentale créée à partir de V5 n'est pas perçue de la même façon que celle qui implique V5 dans une cascade activée à partir de la rétine. Il n'en reste pas moins que le lien ainsi établi entre l'activation d'une région corticale et la création par le cerveau d'une image mentale est un pas dans l'analyse des phénomènes neurobiologiques liés à la conscience. Premier pas... avec les techniques modernes car il y a bien longtemps que des neurologues, étudiant certaines crises épileptiques, avaient révélé de telles associations. A la fin du XIX ${ }^{e}$ siècle, Hughlings Jackson avait décrit, parmi les auras épileptiques, ce qu'il avait appelé le dreamy state (état de rêve) au cours duquel le patient créait une imagerie mentale souvent très riche, proche du rêve. Les études corticographiques de Wilder Penfield et stéréo-encéphalographiques de Jean Talairach et Jean Bancaud ont permis de localiser les décharges épileptiques associées à ce dreamy state dans les régions temporales moyennes et postérieures, c'est-à-dire, de façon fort intéressante, à proximité de V5.

M.P.

\footnotetext{
1. Damasio AR. Knowing how, knowing where Nature 1995; 375: 106.7.

2. Crick $F$, Koch $C$. Are we aware of neural activity in primary visual cortex? Nature 1995; 375: 121 3

3. Tootell RBH, Reppas JB, Dale AM. Visual motion after-effect in human cortical area $M T$ revealed by functional magnetic resonance imaging. Nature 1995; 375 : 139-41.
}

\section{BRÈVES}

Deux pas en avant dans le domaine des greffes intracérébrales de neurones viennent d'être accomplis aux États-Unis. Le premier pas a été réalisé par un groupe collaboratif conduit par Jeff Kordower (Rush Chicago, OH, USA) qui présente l'autopsie d'un patient, greffé pour traitement de la maladie de Parkinson depuis dix-huit mois et décédé d'une maladie indépendante [1]. Les résultats de cette étude sont particulièrement bienvenus en cela qu'ils permettent de clore un certain nombre de controverses nées, justement, de l'absence de données précises chez l'homme permettant de valider les extrapolations faites à partir des études réalisées chez l'animal. Premier point, l'analyse immunocytochimique utilisant des marqueurs des neurones catécholaminergiques révèle l'existence d'une dense réinnervation du tissu hôte émergeant du greffon de neurones fotaux. Cela n'est pas une surprise pour les "greffeurs" expérimentalistes qui ont l'habitude de retrouver $95 \%$ à $100 \%$ de leurs greffons chez l'animal mais les résultats d'autopsies pratiquées précédemment par deux autres groupes - dans des conditions de préservation nettement moins bonnes, il faut le préciser n'avaient pas été aussi positifs. Il faut dire que dans ces deux derniers cas, les greffes n'avaient pas été réalisées suivant les techniques " standards" car elles utilisaient, soit des tissus congelés [2], soit des neurones provenant de fotus "âgés " (plus de 10 semaines de gestation) [3]. Deuxième résultat important, il existait chez ce patient une excellente corrélation entre l'amélioration clinique et la réinnervation striatale, d'une part, entre celle-ci et les images de captage de la fluoro-dopa obtenues en tomographie à émission de positons, d'autre part. ('e dernier point est très important car on ne savait pas bien jusque-là quel crédit apporter à ces images et leur valeur comme test de la réinnervation est donc aujourd'hui validée. Il est à noter, car cela avait été suggéré, que l'innervation catécholaminergique observée provenait bien du greffon et non d'une réinnervation endogène induite par une libération "non spécifique" de facteurs neurotrophiques. Enfin, et il ne s'agit pas d'un point mineur pour les immunologistes, ces greffons provenant de foutus chez lesquels aucun typage HLA n'avait été réalisé - avaient survécu dans le cerveau du patient pendant dix-huit mois dont douze sans aucun traitement immunosuppresseur... Le second pas récemment réalisé par une autre équipe américaine est celui de la xénogreffe. Il n'existe pas, encore, de publication sur le sujet mais la grande presse s'est fait l'écho de la greffe, par l'équipe de Jim Schumacher et d'Ole Isacson de la Harvard Medical School de Boston, de neurones dopaminergiques de porc dans le cerveau de patients parkinsoniens. Il est tout à fait évident qu'une telle expérimentation requiert une analyse approfondie, au moment où les résultats cliniques et biologiques pourront être interprétés, c'est-à-dire pas avant de nombreux mois. Il s'agit toutefois à l'évidence du passage d'une frontière, tant sur le plan biologique - car les résultats expérimentaux obtenus chez l'animal permettent réellement de fonder quelques espoirs quant à la réussite de l'in tervention et au maintien au long cours des neurones greffés - que sur le plan philosophique car la construction d'une telle chimère cérébrale remet en cause nombre de dogmes et elle ne manquera sûrement pas de détracteurs.

[1. Kordower JH, et al. $N$ Engl J Med $1995 ; 332$ : $1118-24$.

[2. Spencer DD, et al. N Engl J Med $1995 ; 327$ : 1541-8.]

[3. Hitchcock EH, et al. Exp Neurol $1994 ; 129$ : 3.] 
Un lien direct entre cassure chromosomique et site fragile du proto-oncogène $C B L 2$. Le syndrome $\mathrm{X}$-fragile est la cause la plus fréquente de retard mental héréditaire. Il est aussi le premier dont la base moléculaire a été définie : expansion d'un trinucléotide (CCG) dans la région 5' du gène $F M R 1$, avec méthylation des îlots Cp(; adjacents et inhibition de la transcription du gène. Outre FRAXA, d'autres sites fragiles ont été trouvés sur le chromosome $\mathrm{X}$, FRAXE, FRAXF $\left(\mathrm{m} / \mathrm{s} n^{\circ} 4\right.$, vol. 10, $p$. 482), seul le premier étant associé à une expression phénotypique $(\mathrm{m} / \mathrm{s}$ $n^{\circ} 4$, vol. 10, p. 472). Un site FRA16A a aussi été mis en évidence sur un autosome, lié au même type de processus. Un travail récent de plusieurs équipes anglaises et australiennes étudie le site fragile rare FRA $11 B$ en l lq23.3 [1]. On a pu localiser ce site au niveau d'un trinucléotide $(C C G)_{n}$ du gène $(: B I .2$, normalement répété onze fois et non polymorphe. Les études familiales ont retrouvé les mêmes caractères que ceux de FRAXA : deux stades sont observés à des générations successives, une prémutation $(\mathrm{n} \leq 100)$ et une amplification qui peut atteindre 700-800 copies. L'hyperméthylation est là aussi un phénomène constant $\left(\mathrm{m} / \mathrm{s} n^{\circ} 8\right.$, vol. 7, p. 877). Une observation particulièrement intéressante a été l'existence dans certaines de ces familles de cas de syndrome de Jacobsen. Ce syndrome, qui associe certaines dysmorphies à un retard mental modéré, a été décrit comme étant dû à la délétion terminale 1 lq23-ter du bras long du chromosome 11. L'étude des haplotypes a confirmé que c'est bien le même chromosome qui présente un site fragile chez certains individus et une délétion chez d'autres ; le chromosome $11^{-}$du sujet présentant un syndrome de Jacobsen est dérivé du chromosome maternel. Le point de cassure est cependant à une distance d'environ $5 \mathrm{cM}$ de $F R A 11 B$. Ce type de cassure à distance a été vu in vitro dans le cas du FRAXA; jamais, cependant, aucun lien direct n'avait été établi in vivo entre un site fragile et une cassure de chromosome. Cette liaison étant inconstante, il semblerait que l'expression d'un phénotype Jacobsen soit fonction du nombre de cellules $1 \mathrm{lq}^{-}$. La question se pose aussi, puisque CBL2 est un proto-oncogène, de l'incidence potentielle de ces réarrangements chromosomiques dans la tumorigenèse.

[1. Jones (;, et al. Nature $1995 ; 376$ : 145-9.]

Chaque épine dendritique est une unité d'intégration fonctionnelle du neurone. Les neurones reçoivent la quasi-totalité de leurs contacts synaptiques au niveau du soma, des dendrites et de très petites excroissances dendritiques que l'on appelle des épines. ('es épines ne sont pas simplement des extensions de la dendrite mais bien des structures spécialisées : elles présentent souvent des morphologies complexes et, notamment, une organisation en deux parties, une tête large et un cou étroit qui les rattachent au tronc dendritique ; elles contiennent aussi un cytosquelette qui possède des caractéristiques propres et un regroupement de polyribosomes. L'importance de ces épines dans la réception des messages synaptiques a été reconnue depuis longtemps en raison, notamment, de leur nombre (30000 sur un neurone pyramidal de CAl dans le cortex hippocampique) et de leur association systématique avec un, voire plusieurs boutons synaptiques. Rafael Yuste et Winfried Denk (AT\&T Labs, New Jersey, USA) démontrent à présent, grâce à une technique d'imagerie du calcium sophistiquée, que les épines dendritiques ne sont pas simplement des sites de réception mais également des sites indépendants d'intégration fonctionnelle dans les neurones [1]. Les auteurs ont utilisé une technique dite de microscopie en fluorescence à double-pho- ton pour étudier les modifications de la concentration intracellulaire du calcium (visualisée grâce à un fluorophore, le calcium-green 1) dans les épines de neurones hippocampiques maintenus dans des tranches, c'est-à-dire dans un environnement cellulaire et synaptique beaucoup plus proche de l'in vivo que dans des cultures. Cette technique s'appuie sur la combinaison d'énergies de deux photons émis à des longueurs d'onde différentes, pour activer le fluorophore grâce à une excitation se situant dans l'infra-rouge, donc très pénétrante, et sous-liminaire, donc peu lésionnelle. Les résultats obtenus démontrent que l'activation d'afférences synaptiques à des niveaux ne provoquant qu'un potentiel post-synaptique d'excitation et pas d'émission de potentiel d'action peut provoquer des accumulations transitoires de calcium spatialement limitées à une ou quelques épines, grâce à l'activation de canaux calciques dépendants du voltage. Les épines dendritiques forment ainsi un " compartiment" à elles seules. Ayant montré par ailleurs que des potentiels d'action émis par une cellule envahissent, de façon "rétrograde", les épines dendritiques de cette même cellule, les auteurs ont observé que la coïncidence d'une activité afférente sous-liminaire et d'un envahissement "rétrograde" provoque un accroissement du calcium intracellulaire supérieur à la somme des augmentations liées à chacun des événements intervenant indépendamment. Ainsi, une épine dendritique "traduit " la coïncidence entre deux phénomènes, l'un pré- et l'autre postsynaptique, par une modification fonctionnelle locale spécifique. La physiologie neuronale paraissait déjà particulièrement complexe jusqu'ici; nous voici avec 30000 sites supplémentaires capables d'une intégration indépendante dans chaque neurone!

[1. Yuste R, Denk W. Nature 1995; 375: 682-4.] 


\section{BRÈVES}

Nous savons bien peu de choses sur les gènes intervenant dans le développement, le contrôle et la répartition de la pilosité chez les humains. La différence marquée existant dans ce domaine entre les hommes et les autres primates laisse supposer qu'à ce stade de l'évolution des modifications importantes sont survenues, soit dans les gènes de régulation, soit dans les gènes de structure. Les personnes atteintes d'hypertrichose ont longtemps été l'objet de curiosité et de crainte, la pilosité étant perçue comme la réapparition d'un caractère perdu au cours de l'évolution : cet "atavisme " amenait à les considérer jadis comme "hommes-singes" ou "hommes-loups". Ils étaient encore montrés dans les foires au siècle dernier. Actuellement, la notion même d'atavisme a changé mais le préjudice esthétique reste considérable, pour les femmes surtout. Indépendamment des fréquentes hypertrichoses d'origine exogène, souvent dues à des déséquilibres hormonaux, il existe de rares cas d'hypertrichose généralisée, généralement transmise sur le mode autosomique dominant. Dans une grande famille mexicaine, suivie sur cinq générations, une pilosité généralisée (CGH, congenital generalized hypertrichosis), épargnant les paumes et les plantes des pieds, et surtout abondante sur le visage et la partie supérieure du corps, a été étudiée d'abord cliniquement [1], puis par analyse de ségrégation [2]. L'arbre généalogique montre clairement une transmission dominante liée à l'X. Dans cette famille, les femmes atteintes ont une distribution irrégulière, conséquence probable de l'inactivation des $\mathrm{X}$ au hasard. L'analyse de 45 marqueurs polymorphes de l'X a permis de situer le gène dans la région $\mathrm{Xq} 24-27$, des recombinaisons ne permettant pas une localisation plus précise. $\mathrm{Si}$ aucune autre famille ne peut être étudiée, une approche par gènes candidats serait la meilleure stratégie. L'existence de syndromes associant diverses anomalies conduit à supposer que les gènes intervenant dans la régulation de la pilosité ont d'autres fonctions. Il peut s'agir de gènes codant pour des facteurs de croissance ou pour leurs récepteurs, des molécules d'adhérence et des enzymes jouant un rôle dans le métabolisme du tissu conjonctif (comme dans la maladie de Hurler par exemple). Pour bien comprendre les hypertrichoses, il faudra d'abord progresser dans la connaissance du développement des follicules pileux [3]. (On sait qu'ils se forment d'abord dans la région céphalique et s'étendent ensuite en ondes à la surface du corps. Un même follicule primaire donne, chez un même sujet, des types de poils différents à des temps de développement différents. De très nombreuses protéines sont synthétisées dans les poils (plus de cent dans les fibres constituant la laine du mouton). Par chance, nous disposons d'une liste impressionnante de mutations du pelage chez la souris qui permettent de nombreuses expérimentations in vivo et in vitro et qui, il faut l'espérer, nous permettront de mieux comprendre la pilosité humaine.

[1. Macias-Flores MA, et al. Hum Genet $1984 ; 66$ : 66-70.]

[2. Figuera LE, et al. Nature Genet $1995 ; 10$ : 202-7.]

[3. Hardy MH. Trends Genet 1992 ; $8: 55-61$.

II n'y a pas que l'art qui compte, il y a aussi la manière. Le calcium joue un rôle essentiel dans le développement neuronal, que l'on a pu mettre en évidence in vitro. Toute variation du calcium extra- ou intracellulaire provoque des modifications dans la morphologie des cônes de croissance, leur orientation ou leur existence même. Par ailleurs, il est bien connu que le calcium module l'activité de multiples protéines, seconds messagers et facteurs de transcription qui participent à un grand nombre de fonctions cellulaires. L'enregistrement des flux cal- ciques dans des neurones en développement a révélé l'existence de courants spontanés de formes différentes, dont les rôles relatifs étaient inconnus. Xiaonan $\mathrm{Gu}$ et Nicolas Sptizer (Univ. California, San Diego, USA) [1] ont utilisé l'imagerie du calcium pour analyser ces phénomènes, ce qui leur a permis d'identifier deux types d'événements spontanés qu'ils ont caractérisés comme des "pointes» et des "ondes". Les pointes sont des courants rapides et intenses alors que les ondes sont des courants plus faibles et plus lents. Comme attendu, la suppression de l'ensemble de ces événements, par culture en milieu dépourvu de calcium, bloque la différenciation et la croissance neuronale. Plus intéressant est le fait que les auteurs ont réussi à associer des aspects différents de la différenciation et de la pousse neuronale à l'un et à l'autre de ces événements en les reproduisant artificiellement, dans des neurones maintenus en milieu sans calcium, par des injections ioniques intracellulaires reproduisant spécifiquement, soit les pointes, soit les ondes. La production de pointes permet aux neurones de se différencier normalement sur le plan chimique et métabolique, mais n'est accompagnée d'aucune modulation de la pousse neuritique par rapport à ce que l'on observe en milieu sans calcium. Au contraire, la production d'ondes calciques intracellulaires induit la pousse neuritique dans les mêmes conditions que lorsque les neurones se développent en milieu avec calcium, sans entraîner de différenciation chimique ou métabolique. La forme (le pattern) des courants calciques dans les cellules paraît donc jouer un rôle essentiel dans le codage de messages différenciateurs portés par le calcium. Il est probable qu'il en est de même dans de nombreuses autres fonctions dans lesquelles le calcium est impliqué, au cours du développement comme chez l'adulte.

[1. (iu X, Spitzer NC. Nature 1995 375: 784-7.] 


\section{BRÈVES}

Un troisième gène de susceptibilité à la maladie d'Alzheimer. I a susceptibilité au développement de la maladie d'Alzheimer apparaît être très souvent gouvernée par des facteurs génétiques. Deux gènes de susceptibilité ont déjà été caractérisés : le gène codant pour la protéine précurseur du peptide $\beta$ amyloïde ( $\beta$ APP) sur le chromosome 21 [1] e t le gène de l'apolipoprotéine E. sur le chromosome $19\left(\mathrm{~m} / \mathrm{s} n^{\circ} 10\right.$, vol. 9, p. 1142). D'autres familles caractérisées par l'apparition d'une maladie d'Alzheimer très précoce, avant 65 ans, semblaient dépendre d'un locus situé sur le bras long du chromosome 14, en 14q24.3. Un consortium international comportant des chercheurs canadiens de Toronto ainsi que des équipes française, américaine, anglaise et italienne vient de parvenir à cloner le gène responsable de cette forme [2]. La méthode de clonage positionnel utilisée est maintenant classique : des études de liaison permettent de raffiner la localisation chromosomique qui est ensuite couverte par un contig de YAC (chromosome artificiel de levure). Des ADN complémentaires sont ensuite sélectionnés sur l'ADN de ces YAC, permettant d'isoler les transcrits de gènes localisés dans cette région. L'un de ces transcrits (\$182) code pour une protéine jusqu'alors inconnue dont la séquence est trouvée modifiée chez les malades. Ce clone correspond à un messager dont la forme majoritaire a une taille de 3 kilobases et dont la séquence permet de prédire qu'il code pour une protéine de 467 acides aminés comportant 7 segments hydrophobes qui pourraient être des régions transmembranaires. La protéine S182 pourrait donc bien être une protéine de membrane, par exemple un récepteur ou un canal. La seule analogie de séquence trouvée dans les banques de données concerne une protéine intervenant dans la spermatogenèse de Caenorhabditis elegans, peut-être en jouant un rôle dans le trafic cellulaire des pro- téines. Si cela vaut également chez l'homme, peut-être peut-on proposer que la protéine Sl82 est capable de régler les transports et la maturation intracellulaire $\mathrm{du}$ peptide $\beta$-amyloïde dont l'accumulation dans les plaques de substance amyloïde est l'un des signes caractéristiques de la maladie d'Alzheimer. Une interaction avec la formation des microtubules et la protéine associée Tau $\left(\mathrm{m} / \mathrm{s} n^{\circ} 4\right.$, rol. $11, p$. 627) peut également être suggérée. Les mutations observées chez les sujets affectés par la maladie sont des mutations faux-sens, aboutissant à la modification d'un acide aminé. Aucune délétion et aucune mutation non-sens ne sont observées. Les acides aminés mutés sont totalement conservés entre la protéine humaine et son homologue murin. Les malades atteints de cette forme familiale d'Alzheimer sont hétérozygotes pour la mutation qui est toujours une modification de la protéine et non pas son absence ou sa troncation importante. On peut donc suggérer que le mécanisme pathogénique est plus vraisemblablement un gain qu'ume perte de fonction. L'expression de la protéine S182 est pratiquement ubiquitaire, comme l'est également celle de la $\beta$ APP. Les interactions fonctionnelles entre cette protéine S182 et les autres éléments de risque que sont le précurseur du peptide amyloïde et l'apolipoprotéine E seront probablement les premières questions que se poseront les spécialistes du domaine à travers le monde.

[1. Dreyfus J. médecine/sciences $1987 ; 3: 254-5$.]

[2. Sherrington $\mathrm{R}$, et al. Nature 1995 ; 375: 754-60.]

L'ARN polymérase, une enzyme qui vient de loin. Les ARN polymérases assurant la transcription du génome nucléaire contiennent de 12 à 16 sous-unités différentes, ce qui les range parmi les enzymes les plus complexes de la cellule. Le clonage des gènes correspondants chez la levure a clarifié l'organisation hétéromultimérique de ces enzymes. Pour chacune des trois polymérases, il existe des sous-unités équivalentes aux chaînes $\beta$, $\beta$ et $\alpha$ de l'enzyme bactérienne, définissant une enzyme minimale qui assure les aspects catalytiques de la transcription. D'autres ne sont présentes que dans une seule des trois enzymes et sont probablement impliquées dans la spécificité transcriptionnelle de l'enzyme correspondante. Enfin, cinq polypeptides de petite taille (de 7 à $27 \mathrm{kDa}$ ) sont partagés par les trois ARN polymérases de levure. TBP, la protéine de liaison à la boîte TATA, est également présente dans les trois complexes de transcription eucaryotes [1]. Le rôle de TBP dans l'assemblage des complexes de transcription est assez bien connu, mais celui des autres protéines communes reste obscur. L'absence d'équivalents bactériens semblait, de prime abord, impliquer des fonctions typiquement eucaryotes, mais on sait depuis peu que TBP a un équivalent structurel chez les archébactéries [2], et que plusieurs sousunités de la polymérase archébactérienne sont semblables aux sous-unités communes de la levure [3]. L.es archébactéries contiennent également des protéines présentant une forte similitude avec les histones, et l'hypothèse d'une structure comparable aux nucléosomes d'organismes supérieurs est donc tentante. Les petites sous-unités communes pourraient, dans ce cas, avoir pour rôle de faciliter la transcription du génome en dépit de cette structuration en nucléosomes Cela expliquerait leur présence commune dans les trois complexes de transcription eucaryotes et leur absence chez les bactéries classiques dont le génome est directement accessible à l'ARN polymérase. Chez l'homme, une collaboration entre des équipes de l'IGBMC à Strasbourg et du CEA-Saclay a récemment montré l'existence de cinq ADNc codant pour des protéines semblables aux sous-unités com- 
munes de la levure. En outre, quatre de ces ADNc sont capables de corriger in vivo le phénotype létal de mutants inactivés pour les sous-unités correspondantes de levure [4] Les sous-unités humaines s'incorporent donc efficacement dans les trois polymérases de levure, malgré la structure hétéromultimérique particulièrement complexe de ces enzymes. (Cette interchangeabilité fonctionnelle ouvre une piste intéressante dans l'étude de la machinerie de transcription chez l'homme et illustre, si besoin en était, l'importance de la levure comme "tube à essai biologique" dans l'étude des grandes fonctions cellulaires chez les eucaryotes

[1. Hernandez N. Genes Dev 1993 ; 7 : 1291-308.]

[2. Rowlands T, et al. Science 1994 ; 264 : 1326-9.]

[3. Langer D, et al. Proc Natl Acad Sci USA 1995 (sous presse).]

[4. Shpakovski GV, et al. Mol Ciell Biol 1995 (sous presse).]

Le récepteur de la dioxine et le complexe transcriptionnel répondant à l'hypoxie appartiennent à la même famille et ont des sous-unités communes. Les gènes codant pour les cytochromes P450 inductibles par les xénobiotiques possèdent un élément de réponse fixant un complexe récepteur connu sous le nom de récepteur de la dioxine ou des xénobiotiques. Il s'agit d'un hétérodimère composé d'une sous-unité AHR (aryl hydrocarbon receptor) et d'une sous-unité ARNT (aryl hydro carbon receptor nuclear translocator), qui appartiennent à la famille des facteurs basique-hélice/boucle/hélice (b-HLH pour basic helix/loop/helix). De plus, ces protéines AHR et ARNT contiennent des domaines conservés, connus sous le nom de PAS pour Per-ARNT-AHR-Sim. Huang et al, de Baltimore (MD, USA) [1] travaillent quant à eux sur un système tout à fait différent : la régulation transcriptionnelle par l'hypoxie. Dans des conditions d'hypoxie, le gène de l'érythropoïétine est induit transcriptionnellement, de même que plusieurs gènes glycolytiques permettant une adaptation métabolique à la diminution de la concentration en oxygène. Le gène de l'érythropoïétine contient un élément de régulation de type enhancer en 3' du gène, fixant un complexe protéique HIF-l (hypoxiainducible factor-1) dont l'activité de liaison augmente dans les conditions d'hypoxie [2]. L'équipe de Baltimore a purifié par des moyens biochimiques le complexe protéique HIF-1 et procédé à la détermination partielle de sa séquence en acides aminés d'où a été déduite la séquence de la sonde oligonucléotidique utilisée pour isoler les ADN complémentaires correspondants. HIF-1 est formée de deux types de sous-unités, HIF-l $\alpha$ et HIF$1 \beta$. HIF-1 $\beta$ existe sous plusieurs isoformes qui correspondent à la molécule ARNT présentée plus haut. HIF-1 $\alpha$ semble appartenir à la même famille des protéines $\beta$-HLHPAS, ayant quelques analogies avec AHR, Sim, etc. Il semble, par conséquent, que HIF-l et le récepteur de la dioxine soient tous deux des hétérodimères de protéines de type $\beta$-HLH-Pas, partageant une sousunité commune HIF-1 $\beta / A R N T$. La régulation du récepteur de la dioxine est avant tout post-transcriptionnelle, par formation de complexes avec les xénobiotiques. La pression partielle en oxygène semble contrôler le taux de synthèse du complexe HIF-1, au moins en partie, par accumulation des ARN messagers en situation d'hypoxie ainsi qu'en présence de chlorure de cobalt, un inducteur connu de l'érythropoïétine. Cependant, de nombreuses séquences potentiellement phosphorylables des sous-unités HIF-1 suggèrent qu'elles pourraient également être soumises à une régulation post-transcriptionnelle.

[1. Huang GL, et al. Proc Natl Acad Sci USA 1995; 92 : 5510-4.]

[2. Lacombe C, Mayeux P. médecine/sciences $1995 ; 11$ : 947-55.]
Le facteur de transcription NF-E2 est indispensable aux stades ultimes de la plaquettogenèse. Les lignées érythroïdes et mégacaryocytaires sont dérivées de précurseurs communs, ce qui explique probablement que les érythroblastes et les mégacaryocytes possèdent des facteurs de transcription en partie identiques : par exemple, GATA-1, NF-E2 et Tal-1/SCL sont exprimés dans les deux types cellulaires. Le facteur de transcription NF-E2 est formé d'un hétérodimère composé d'une sous-unité hématopoïétique p45 et d'une sous-unité ubiquitaire p18. Des sites NF-E2 et GATA-1 sont retrouvés dans les régions régulatrices de nombreux gènes érythroïdes, notamment les gènes de globine. Plusieurs groupes américains associés, collaborant avec la firme AMGEN, viennent de rapporter les conséquences de l'invalidation du gène $N F-E 2$ par recombinaison homologue [1]. Les souris $\mathrm{NF}-\mathrm{E} 2^{-/-}$ont une érythropoï̀se sensiblement normale mais meurent en grand nombre précocement d'un syndrome hémorragique en rapport avec une absence de plaquettes sanguines. Cependant, les mégacaryocytes sont présents dans la moelle et leur réponse proliférative à la thrombopoïétine $\left(\mathrm{m} / \mathrm{s} n^{\circ} 8-9\right.$, vol. 10, p.874) est normale. D'ailleurs, la concentration de thrombopoïétine circulante est également sensiblement normale, malgré l'absence de plaquettes sanguines. Ces résultats indiquent que le facteur NF-E2, quoique se liant à de très nombreuses régions régulatrices de gènes exprimés dans les érythroblastes, joue un rôle plus important dans la thrombopoïèse que dans l'érythropoïèse. NF-E2 semble particulièrement indispensable pour la maturation tardive des mégacaryocytes et le phénomène de plaquettogenèse caractérisé par la fragmentation des mégacaryocytes polyploïdes en de multiples plaquettes. La thrombopoiétine, quant à elle, pourrait intervenir à de nombreux niveaux et, en ce qui concerne la lignée mégacaryocytaire, sur la 
différenciation et la prolifération de ces cellules. L'absence d'élévation de thrombopoïétine circulante malgré l'absence de plaquettes suggère que le stimulus de l'augmentation de la thrombopoḯtine pourrait ne pas être la thrombopénic elle-même mais plutôt le nombre de mégacaryocytes ou la libération de facteurs d'origine plaquettaire en cas de purpura thrombopénique d'origine immunologique.

[1. Shivdasani RA, et al. Cell 1995 ; 81 : 695-704]

Déponse adaptative au paludisme à $P$. vivax : la mutation spécifique, dans le globule rouge, de l'antigène qui en serait le récepteur. En 1993, la résistance fréquente des populations noires à l'infestation palustre par le Plasmodium vivax recevait une base moléculaire : la majorité de ces populations sont Duiffy-négatives, leurs érythrocytes ne fixent pas le $l$. vivax ni les chemokines dont l'antigène 1$) u f f y$ est le récepteur habituel $\left(m / s n^{\circ} 11\right.$, vol 9 , p. 1265). Le clonage du gène codant pour l'antigène Duffy a permis des avancées importantes dans la compréhension des mécanismes impliqués. Un polymorphisme Fya/Fyb permet d'identifier des sujets Fy $(a+b+), F y(a+b-)$ et Fy $(a-b+)$, en dehors même des sujets I)uffy-négatifs qui sont $\mathrm{Fy}(\mathrm{a}-\mathrm{b}-)$. La base moléculaire de ce polymorphisme, identifiée par trois groupes, simultanément en France, aux Etats-Unis et a u Japon [1-3], est une mutation ponctuelle $\mathrm{G} \rightarrow \mathrm{A}$, traduite au niveau protéique par une substitution Gly $\rightarrow$ Asp, correspondant, respectivement, aux allèles $F Y^{*} A$ et $F Y^{*} B$. Ce dernier allèle est le plus fréquent dans les populations noires. L'étude du gène I)ARC (I)uffy antigen/chemokin receptor) a été poursuivic à Paris dans l'équipe de Cartron [4]. Il s'agit d'un gène de petite taille ne comportant qu'un seul exon. Par ailleurs, le transcrit de ce gène DARC, absent dans la moelle osseuse des sujets Duffynégatifs, est largement exprimé dans d'autres tissus : rein, rate, foic fotal, cerveau, cellules endothéliales veineuses. N'ayant pu identifier aucune mutation dans la séquence codante du gène, les auteurs ont fait l'hypothèse d'unc régulation spécifique propre aux tissus érythroïdes et analysé dans cette perspective la région promotrice. Parmi les séquences consensus retrouvées, se trouvent deux séquences GATA, caractéristiques de tous les gènes à expression érythroïde. Chez les sujets Fy(a-b-), une de ces séquences est interrompue par une mutation $\mathrm{T} \rightarrow \mathrm{C}$ en -46 ; l'absence de fixation du facteur GATA sur la séquence mutée a pu être confirméc expérimentalement. Il s'agit donc du premier exemple de la répression spécifique de tissu d'un gène, par ailleurs largement exprimé, par la mutation d'un élément régulateur en cis, lui aussi spécifique d'un tissu. L'amplification sélective che\% les Noirs d'Afrique de l'Ouest d'un allèle $F Y$, mutant de l'allèle $F Y^{*} B$, peut sans doute être considérée comme une réponse adaptative de résistance au paludisme.

[1. Tourmamille (', et al. Hum Genet $1995 ; 95: 407-10$.

[2. Chaudhuri A, et al. Blood 1995 ; 85 : 15-21.]

[3. Iwamoto S, et al. Blood $1995 ; 85$ : 622-6.]

[4. Tourmamille (', et al. Nature (ienet $1995 ; 10: 224-8$.]

Un gène, une queue. La mobilité cellulaire est un aspect essenticl de la physiologie de nombreuses cellules eucaryotes et procaryotes. C'est ainsi que l'augmentation de la mobilité des cellules tumorales joue un rôle dans leur invasivité et propension à métastaser. Chez les bactéries, la mobilité est souvent un élément important de virulence. Ainsi, Shigella flexneri et Lysteria monocytogenes sont-ils mus par une sorte de queue postérieure dans laquelle sont arrangées des fibres d'actine polymérisées. L'équipe de Philippe Sansonetti avait démontré, il y a environ six ans, que les shigelles déficientes dans l'expression du gène Ics $A$ étaient dépourvues de queue et de mobilité. Maintenant, les équipes de Julie Theriot (Cambridge, MA, USA) et Marcia Goldberg (New York, USA) [1] montrent que le transfert du gène Ics $A$ dans E.coli suffit à faire apparaître dans cet organisme une queue d'actine lui permettant de se mouvoir aussi rapidement que les shigelles, c'est-à-dire d'environ $1 \mathrm{~mm}$ par heure. Cela signifie que la seule fonction nécessaire à la morphogenèse des queues d'actine est la fonction IcsA. Il est possible, néanmoins, que la protéine lcsA interagisse avec d'autres protéines communes aux shigelles et aux colibacilles.

D'autres shigelles que $S$. flexneri sont responsables des dysenteries épidémiques entraînant la mort de milliers de malades tous les ans à travers le monde. Le développement d'une résistance aux antibiotiques de ces agents infectieux et les conditions propres des pays du tiers monde rendent tout à fait souhaitable la mise au point d'une vaccination contre ces dysenteries. Un mutant de shigelle, immobile à la suite d'une mutation du gène IcsA et atténué par une autre modification génétique, pourrait constituer la base d'un vaccin vivant inoffensif. Les premiers essais de phase 1 ont été commencés en juillet 1995 che\% l'homme.

[1. Goldberg MB, Theriot J. Pror Natl Acad Sci USA 1995 ; 92 : 6572-6.]

Effet des oestrogènes sur l'érythropoièse par modulation de l'action d'un facteur de transcription spécifique, GATA-1. Les hormones stéroïdes, et les œestrogènes en particulier, font partie des facteurs intervenant dans la régulation 
de l'hématopoïèse. On sait en effet que les ostrogènes induisent une anémie chez les mammifères ; chez le poulet, on a observé que la maturation des cellules progénitrices est inhibée malgré leur prolifération, en même temps qu'une régulation négative de nombreux gènes érythroïdes est constante. Un travail récent de l'équipe de S.H. Orkin a pu préciser les mécanismes en cause dans ces faits d'observation [1]. Les auteurs ont d'abord observé en culture primaire de moelle la même inhibition de la différenciation érythroïde des progéniteurs en BFU-E (burst forming units, erythroid). Sachant le rôle prépondérant du facteur de transcription GATA-1 une protéine à doigts de zinc $(\mathrm{m} / \mathrm{s}$ $n^{\circ} 4$, vol. $7, p$. 385) sur la différenciation érythroïde et l'expression de nombreux gènes spécifiques, ils ont envisagé GATA-1 comme une cible possible de l'action des œstrogènes. On sait, en effet, que les hormones stéroïdes interagissent avec leurs récepteurs, mais aussi avec d'autres facteurs de transcription. Comme on ne trouve pas de site de liaison pour le récepteur d'œstrogène (ER) dans les promoteurs érythroïdes, on peut donc penser à une interaction fonctionnelle avec GATA-1. La démonstration a procédé par plusieurs étapes. L'addition d'œestrogène et de son récepteur réduit drastiquement (10 fois) la trans-activation par GATA-1 d'un promoteur artificiel dont la sensibilité à ce facteur est connue ; cet effet est inversé par un antiœstrogène, ce qui est en faveur de l'interaction directe entre ER qui a fixé le ligand et GATA-1, et non d'un simple effet de toxicité. Le même effet d'inhibition est retrouvé sur un promoteur naturel intact. L'interaction des deux protéines et la formation d'un complexe ER/GATA-1 a été démontrée in vitro en l'absence d'ADN. In vivo, dans des cultures de cellules COS transfectées, l'addition d'un anticorps monoclonal provoque également la co-immunoprécipitation du complexe. Il existe donc entre les deux protéines des contacts directs, que les auteurs ont ensuite cherché à localiser. La fixation de l'ensemble ER-ligand a été testée sur différentes constructions de GATA-1, ce qui a permis de démontrer qu'une interaction fonctionnelle nécessite deux contacts au moins, l'un dans la région des doigts de zinc, l'autre à l'extrémité N-terminale. L'ensemble de ces résultats semble expliquer l'effet des astrogènes sur l'érythropoïèse par une activité modulatrice de type protéine/protéine exercée par le récepteur ER sur GATA-1. Peut-on envisager que d'autres stéroïdes agissent sur la différenciation par interférence avec d'autres facteurs GATA?

[1. Blobel GA, et al. Mol Cell Biol $1995 ; 15$ : 3147-53.]

Un possible mécanisme de la résistance au tamoxifène des cancers du sein : une phosphorylation activatrice du récepteur des oestrogènes. Les oestrogènes sont des facteurs de croissance pour les cellules mammaires, justifiant le traitement des cancers du sein par des antiœstrogènes de la famille du tamoxifène. Cependant, certaines tumeurs résistantes au tamoxifène sont négatives pour la présence du récepteur des oestrogènes (RE) et possèdent, très souvent associée, une amplification du gène codant pour le récepteur membranaire à activité de tyrosine kinase HER-2, également connu sous le nom de erbB2 ou Neu $\left(m / s n^{\circ} 7\right.$, vol. 5, p. 514). Ce récepteur a comme ligand des peptides appelés hérégulines $\left(\mathrm{m} / \mathrm{s} n^{\circ} 7\right.$, vol. 8, p. 734). Pietras et al. du laboratoire de Dennis Slamon (Los Angeles, CA, USA) [1], démontrent dans un récent article d'Oncogene qu'il peut exister une interaction directe entre ce système de récepteur/ligands et le récepteur des œstrogènes. En effet, la transfection dans des cellules tumorales mammaires en culture MCF7 de vecteurs d'expression de l'héréguline ou du récepteur HER-2 aboutit à la phosphorylation du RE et à l'activation du gène codant pour le récepteur de la progestérone, une cible des œestrogènes. Ces résultats suggèrent très fortement que l'hyperexpression d'HER-2, ou bien son activation par la liaison des ligands hérégulines, aboutit à la phosphorylation et à l'activation du $\mathrm{RE}$, même en l'absence d'œestrogènes. Il est probable que le RE ainsi phosphorylé sur un résidu tyrosine peut se fixer à l'ERE (estrogen response ele ment) en l'absence de l'hormone. Le tamoxifène, qui est un antagoniste partiel du récepteur, devient sans effet alors que d'autres inhibiteurs anti-œstrogènes purs développés par la firme ICI bloquent l'effet œestrogénique de la phosphorylation du RE. Les produits ICI bloquent la dimérisation du récepteur modifient son taux de renouvellement et empêchent, contrairement au tamoxifène, sa fixation à l'ERE. Il est probable que cet effet se manifeste également à l'encontre du RE phosphorylé par le récepteur tyrosine kinase HER-2. L'activation du RE aboutit non seulement à l'augmentation de la transcription du gène du récepteur de la progestérone, mais également à l'inhibition de la transcription du gène du RE luimême, ce qui pourrait expliquer la fréquence avec laquelle les tumeurs mammaires de phénotype HER-2 amplifié sont négatives pour le récepteur des ostrogènes et celui de la progestérone.

[1. Pietras RJ, et al. Oncogene 1995 ; 10: 2435-46.]

D Des syndromes thalassémiques dus à la mutation d'un gène de régulation ? Nombreuses ont été ces dernières années les études systématiques de $\beta$-thalassémies dans différents pays ; quelques cas non identifiés et sans explication se retrouvent dans presque toutes les séries, quelle que soit la qualité des techniques employées. Un travail 
récent, mené de façon indépendante par deux équipes, celle de F. Grosveld à Rotterdam [1] et celle de S.H. Orkin à Boston [2], introduit dans ce domaine une hypothèse nouvelle. Parmi les facteurs de transcription qui règlent l'expression des gènes du locus $\beta$-globine, le facteur EKLF (erythroid krüppel-like factor) semble se lier de façon spécifique à la séquence CACCC située dans le promoteur du gène adulte $\beta$-globine ; il interviendrait dans l'interaction entre le LCR (locus control region) et ce gène et pourrait de ce fait avoir un rôle au cours du développement. Le gène codant pour ce facteur a été inactivé chez la souris, par insertion d'un gène rapporteur dans un cas, par recombinaison homologue dans l'autre, et l'étude du développement des cellules embryonnaires a été observée au cours du développement de l'animal. On a ainsi pu constater que les animaux $\mathrm{EKLF}^{+/-}$se développent normalement. Les animaux $\mathrm{EKLF}^{-/-}$, en revanche, présentent un développement normal pendant la vie embryonnaire, mais, au moment de la transition de l'érythropoïèse du sac vitellin vers le foie, ils développent une anémie d'évolution mortelle, et on voit apparaître, à côté des érythrocytes embryonnaires, d'autres cellules morphologiquement anormales dont l'hémoglobinisation se fait mal. Les images observées sont très comparables à ce que l'on voit au cours des thalassémies majeures chez l'homme. Une différence notable est que ce défaut d'hémoglobinisation n'apparaît chez l'homme qu'au moment de la transition de l'hémoglobine fotale vers l'hémoglobine adulte ; mais il n'existe chez la souris qu'une seule commutation de l'érythropoïèse embryonnaire à l'érythropoïèse fœtale/adulte. Les résultats de ces explorations semblent cependant montrer que le facteur EKLF serait essentiel pour les dernières étapes de l'érythropoïèse définitive. Des mutations inactivant l'expression du facteur EKLF chez l'homme pour- raient donc être à l'origine de syndromes thalassémiques sévères, plus sévères même que les mutations des séquences CACCC qui sont leur cible naturelle.

[1. Nuez B, et al. Nature 1995; 375 : 316-8.]

[2. Perkins AC, et al. Nature 1995; 375 : 318-22.]

$\square$ La protéine Rb est un inhibiteur transcriptionnel ciblé par E2F. La protéine $\mathrm{p} 105^{R h}$, produit du gène de susceptibilité au rétinoblastome, forme des complexes avec le facteur transcriptionnel E2F lorsqu'elle est déphosphorylée, inhibant l'effet de $\mathrm{E} 2 \mathrm{~F}$ sur les gènes indispensables à la prolifération cellulaire. Weintraub et al. (Saint Louis, MO, USA) montrent que la protéine $\mathrm{Rb}$ peut se fixer à l'ADN par l'intermédiaire de E2F, et qu'elle bloque alors l'effet des facteurs transcriptionnels activateurs environnants, probablement en inhibant leurs interactions avec le complexe de base de la transcription. Le même effet peut être obtenu lorsque, en l'absence de site de fixation de E2F, on transfère dans la cellule un vecteur d'expression pour une protéine hybride Rb/GAL4 pouvant se fixer sur un site ADN de reconnaissance du facteur GAL4. L'activité répressive de $\mathrm{Rb}$ fixé à proximité des complexes transcriptionnels est forte puisqu'elle bloque le pouvoir activateur de enhancers viraux, tel celui du virus SV40.

[1. Weintraub SJ, et al. Nature 1995 ; $375: 812-5$.]

L'échange direct de molécules informatives entre les cellules corticosurrénaliennes via les jonctions communicantes augmente la sensibilité de ces cellules à l'ACTH. La réponse biologique d'un organe est à chaque instant le résultat de l'activité "intégrée" d'un ensemble de cellules qui adaptent leur réponse aux besoins de l'organisme. Cette coordination fait intervenir des facteurs extracellulaires auxquels chaque cellule a sa propre sensibilité. Des échanges directs de molécules entre cellules adjacentes peuvent participer à cette synchronisation. Ces échanges ont lieu via des structures spécialisées de la membrane plasmique, les jonctions communicantes ou gap jonctions (GJ). Les GJ sont formées d'un grand nombre de canaux transmembranaires mettant en contact direct le cytoplasme de cellules voisines. Ces canaux permettent le passage de molécules hydrophiles diverses de masse inférieure à 1000 Da. Parmi ces molécules figurent les seconds messagers $\left(\mathrm{Ca}^{2+}\right.$, IP3, AMPc...). L'implication réelle de tels échanges dans une situation physiologique a rarement été démontrée. Dans les glandes surrénales, les cellules de la zone fasciculo-réticulée sont connectées par des GJ. Les auteurs [1] ont montré par micro-injection cellulaire d'une sonde fluorescente, le jaune de Lucif er, que les cellules corticosurrénaliennes d'origine humaine et bovine sont fortement couplées via ce type de canaux en culture primaire. Ils ont étudié l'impact fonctionnel de ce couplage sur l'activité stéroïdogénique de ces cellules en réponse à l'ACTH. En utilisant un composé qui provoque la fermeture rapide et réversible des canaux des GJ, l'acide $18 \alpha$ glycyrrhétinique (18- $\alpha \mathrm{GA})$, les auteurs ont observé une diminution de la production de cortisol induite par des doses sousmaximales d'ACTH. La production basale de cortisol ainsi que la production de cortisol induite par une dose maximale d'ACTH ne sont pas affectées par la fermeture des canaux. En présence de l'inhibiteur, il faut 25 fois plus d'ACTH pour obtenir la demi-stimulation maximale (ED50) de la production de cortisol. Le 18- $\alpha \mathrm{GA}$ ne modifie pas la production de cortisol induite par le 8Br-AMPc qui est un analogue structural du messager intracellulaire de l'ACTH, l'AMPc. Des 


\section{BRÈVES}

contrôles ont été effectués montrant que le 18 $\alpha$-GA n'affecte ni la liaison de l'ACTH à ses récepteurs, ni la production d'AMPc induite par l'ÁCTH. Ces résultats démontrent que des échanges de molécules informatives ont lieu via les $\mathrm{GJ}$ reliant des cellules répondeuses et des cellules non répondeuses à l'ACTH. Par ce phénomène qui a lieu à des doses "physiologiques" d'ACTH $\left(10^{-12}\right.$ à $\left.10^{-10} \mathrm{M}\right)$, les cellules corticosurrénaliennes augmentent leur sensibilité à cette hormone.

[1. Munari-Silem Y, et al. J Clin Invest 1995 ; 95 : 1429- 39.] 